\title{
Adherence to new Royal College of Ophthalmology guidance for hydroxychloroquine retinal screening in rheumatology patients
}

\author{
Authors: Christopher Uy, ${ }^{A}$ Mohammed Iqbal, ${ }^{B}$ Sophiya Thapa ${ }^{B}$ and Taryn Youngstein ${ }^{B}$
}

\section{Introduction}

In February 2018, the Royal College of Ophthalmology (RCO) changed its guidance on screening for retinal toxicity in those prescribed hydroxychloroquine (HCQ). ${ }^{1}$ This was a marked change in guidelines from annual optician review to baseline screening by an ophthalmologist with optical coherence tomography (OCT) to scan the macula within the first 12 months of starting the drug or to create a new baseline for those taking the medication long term. Annual screening was also newly recommended annually after 5 years of treatment.

This change was based on emerging evidence that HCQ retinopathy is more common than previously considered. Reported prevalence is approximately $7.5 \%$ at 5 years on standard dosing (200 mg twice daily) and increases up to $>20 \%$ after 20 years of treatment. ${ }^{2}$ Known major risk factors for HCQ retinopathy are duration of treatment, high dose by weight, concomitant tamoxifen use, known retinal or macular disease, and renal impairment (Table 1). ${ }^{3}$ The change in screening standard was not resourced and initial concerns were raised by ophthalmologists, rheumatologists, nephrologists and dermatologists - the most frequent prescribers of HCQ. Nevertheless, locally we agreed to adopt the guidance, where possible, and referral from primary care (the prescribers) was the locally agreed pathway.

We sought to assess at 1 year whether these recommendations have been implemented and documented in and estimate the prevalence of HCQ retinopathy in the rheumatology outpatient cohort.

\section{Materials and methods}

The notes of 347 patients prescribed HCQ by the rheumatology department were reviewed from 2018-2019. Clinic letters were reviewed for documented HCQ start date, ophthalmology referral (direct or from primary care), eye symptoms and diagnosis of retinopathy. Simple univariate analysis was used to analyse the data with Excel (Microsoft) and jamovi (The jamovi project).

Authors: ${ }^{\mathrm{A}}$ East Sussex Healthcare NHS Trust, London, UK; ${ }^{\mathrm{B}}$ Imperial College Healthcare NHS Trust, London, UK

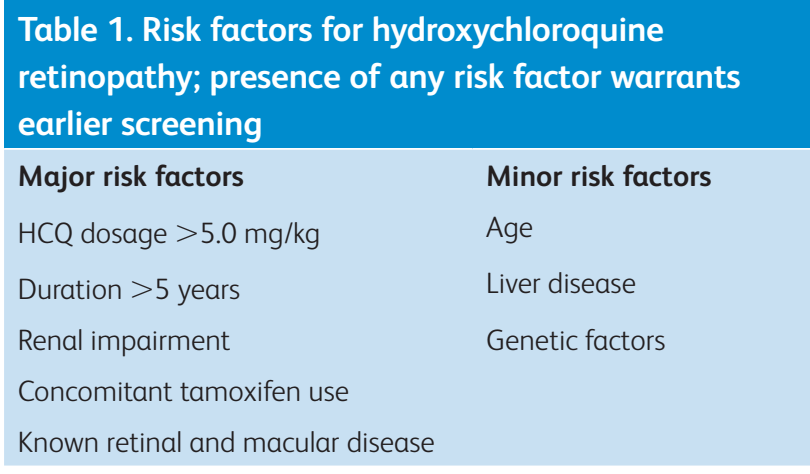

\section{Results and discussion}

Median duration on HCQ was 3.59 years (range 0.00-24.64; Fig 1); $34.9 \%$ of patients were on HCQ for $\geq 5$ years; 254 $(73.60 \%)$ of patients had been referred to ophthalmology,

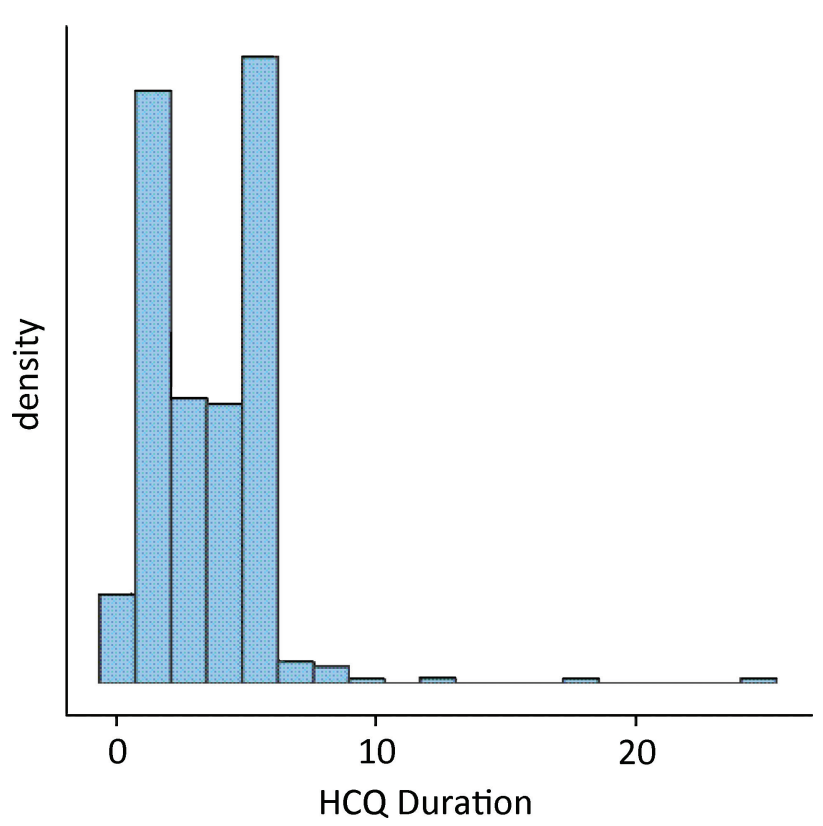

Fig 1. Frequency of patients by duration in years. 
but 92 patients $(26.5 \%)$ of these patients did not mention ophthalmology review in the general practitioner clinic correspondence; 22 individuals (6\%) experienced documented new visual symptoms; six patients $(2 \%)$ had diagnosed retinopathy; two cases $(0.67 \%)$ were confirmed to have HCQ retinopathy. There was a lower documented referral rate for patients on HCQ $\geq 5$ years (high risk group) of only $68.60 \%$ vs $76.33 \%$ but this was not statistically significant (chi-squared $\mathrm{p}=0.119)$.

\section{Conclusions}

Local practice has adopted the 2018 guideline for ophthalmology review and complied with documentation in $73.5 \%$ of patients at baseline. The remaining $26.5 \%$ of identified patients had a letter sent to their general practitioners recommending ophthalmologic screening. Future challenges include the identification of further risk factors for the development of retinopathy, using serum drug levels to allow dose optimisation to avoid over treatment, and to establish mobile HCQ screening clinics alongside rheumatology clinics.

\section{Conflicts of interest}

None declared.

\section{References}

1 Royal College of Ophthalmologists. Hydroxychloroquine and chloroquine retinopathy: recommendations on screening. RCO, 2018. www.rcophth.ac.uk/wp-content/uploads/2018/07/ Hydroxychloroquine-and-Chloroquine-Retinopathy-ScreeningGuideline-Recommendations.pdf [Accessed 18 August 2019].

2 Melles R, Marmor M. The risk of toxic retinopathy in patients on long-term hydroxychloroquine therapy. JAMA Ophthalmology 2014;132;1453.

3 Marmor M, Kellner U, Lai T, Melles R, Mieler W. Recommendations on Screening for Chloroquine and Hydroxychloroquine Retinopathy (2016 Revision). Ophthalmology 2016;123:1386-94. 